Aus der Chirurgischen Universitätsklinik zu Kiel. (Direktor:

Geheimrat Prof. Dr. Anschutz.l

\title{
Zur Frage des Wurmfortsatzdivertikels und seiner Folgezustände, insbesondere des Pseudomyxoma peritonei.
}

Von Dr. W. Löhr, Assistent der Klınkk.

Mit 7 Abbildungen.

In der riesigen Literatur uber Appendicitis und deren Folgezustände ist die Zahl der Mitteïungen uber das Wurmfortsatzdivertikel und seine Folgezustände gering. Es handelt sich im wesentlichen um kasuistische Nittellungen. Ein klinisch zusammenhängendes Bild dieser Erkrankung wurde bisher nicht gegeben. Dagegen ist die Genese der Wurmfortsatzdivertikel pathologisch-anatomisch so geklärt, daß Neues kaum mehr gebracht werden durfte. In der Mehrzahl der mitgeteilten Fälle - übrigens kennt man Appendix-Divertikel erst seit rund zo Jahren — handelt es sich um kleine Divertikel, die gelegentlich von Operationen und Autopsien gefunden wurden. Wenn wir schlechthin von Appendixdivertikeln reden, so meinen wir sog. .,falsche Divertikel“. Bisher wurde nur von Hedinger ein ,echtes" Divertikel beschrieben. v. Brunn ist der erste, der 1905 ein übersichtliches Bild der Pathogenese der Divertikelbildung auf Grund ron 8 eigenen Fällen gibt. Nach ihm gehört das Divertikel des Wurmfortsatzes durchaus nicht zu den Seltenheiten. In ganz überwiegender Mehrzahl entsteht es durch eine vorausgegangene Entzündung des Wurmfortsatzes, welche zu einer Zerstorung der ganzen Wanddicke oder eines Teiles der Wandschichten, insbesondere der Muscularis geführt hat. An der Bildung der Narbe beteiligen sich hauptsächlich die Submucosa und Subserosa. I909 finden v. Brunns Forschungsergebnisse in dem Referat Oberndorfers in Lubarsch-Ostertags Ergebnissen eine vollkommene 
Anerkennung. Die v. Brumsche Erklärung der Divertikelbildung als Folgeerscheinung der Appendicitis erhält auch eine Stütze durch die Arbeiten Aschoffs uber die Appendicitis, in denen nachgewiesen wird, daß un einer großen Anzahl won Fällen den Krypten der Wurmfortsatztonsillen entuprechend eine umschriebene Entzundung oder doch sehr starke Leukocytenanhàufung in der Nuscularis auftritt, die diecelbe schon zum Einschmelzen bringen kann, während die Schlemhaut noch fast unverändert ist und ungeschäligt bleibt. Es konnen so Bilder entutehen, bes denen die uneranderte Nucosit und Submucona durch die durch Entzundung entstandene Muskellucke nach außen rorgewölbt werden. Während num oberndorfer, Lejars und Méné trier, r. Brunn usw. allein der entzundlichen Genese des Wurmfortsatzdivertikels das Wort reden, so liegen doch auch zweifellos Beobachtungen ron Dhertikelbildumgen vor, die durch Einsinken von Schleimhat in praformierte Munkellücken (Grasersche Enissarien), de bei der Einmundung der senkrecht zur Appendixachse verlaufenden (icfialse setroffen werden, entstehen. Derartige Divertikel ind am ubrigen Darm durchau molhts I'n-

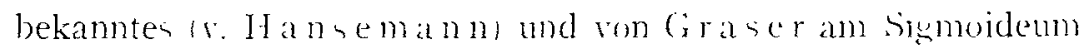
I 890 ) bereit congehend beschrithen. Sulche seltenen Dwertikel

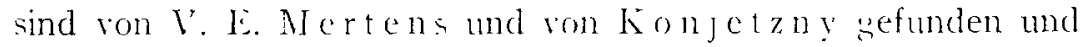
mitgeteilt.

Es gibt also I)iscrtikel auf entzundlicher und nicht entzündlicher Basis. In ganz uberwiegender Menge legen der Divertıkelbildung allerdings entzundliche Prozesse in der Muscularis im Sinne v. Brunng und vor allem . Ischoffs zugrunde. Unter Iooo von Kon jet $\angle n y$ in unserer Klnik untersuchlen tppendices fanden sich 2 Divertikel auf nicht entzundlicher, $5 \mathrm{mtl}$ entzundlicher Genese. Bet 300 darauthn untersuchten . Appendices fand ich + mai Divertikelbildung entzundlicher Bildungsart. Eine Anzahl Appendices wiesen an den Einmundungsstellen der Gefäße leichte Schlemblautemsenkungen auf. Vorutufen der Divertikelbildung nicht entzündlicher Art.

Aus der verschiedenen Art der (ienese der Appendixdivertikelbildung ist somit der Schlulis berechtigt, dal de auf nicht entzündlicher Basis entstandenen Divertikel auch klinısch symptomlos vorlaufen koment. Ion den betden Kimjetznychen fällen war 
der eine gelegentlich einer andersartigen Operation mit entfernt --- von dem zweiten liegt leider kein Krankenbericht vor - : cbenfalls sind die beiden in Frage kommenden Iälle ron Mert e $n$ s gelegentlich ron Autopsien im Nauwerkschen Institut zufällig gefunden.

Dic klinischen Erscheinungen der Divertikel entzündlicher Entstehungsart sind mit den Erscheinungen der rezidivierenden Appendicitis derart eng rerbunden, daß uns auch nach Einsicht in die einschlägige Literatur die Aborenzung eines besonderen klinischen Krankheitsbildes ron dem ler chronischen rezidivierenden Appendicitss unmöglich erschemt. So kommt es wohl auch. dals z. B. Sprengel in seiner . bhandlung über Appendicitis der Divertikel Erwähnung tut.

Pathologisch-anatomisch betrachtet handelt es sich bei der cntzündlichen Divertikelbildung sogar um einen ,eigenartigen Heilungsvorgang" (v. Brunn). Daß aber das ausgebildete Divertikel leicht zu neuen Infektionen den Boden abgibt, ist ohne weiteres verständlich. Demzufolge finden wir in der Literatur in der uberwiegenden Anzahl der niedergelegten Beiträge die akut entzundlichen Erscheinungen betont (vgl. El be, Simon); nicht un. beträhtlich ist aber die Zahl der falle, wo nach .Aunheilumg." der Appendicitis mit Divertikelbuldung auch klinisch dauend ein Ruhezustand eingetreten ist.

Die äußere Form der Divertikel ist entsprechend der Größe der Muskellücke rerschieden. Oft findet man Zwerchsackform. deren Wandung ron mehr oder weniger entzundlicher Mucosa und Subserosa und jüngeren oder älteren Schwartenbildungen begrenzt wird Auf Querschnitten durch die Appendix und ihren Divertikelanhang hat man oft den Findruck, als ob zwei Appendices nebeneinander lagen. Das Tipithel der Mucosa im Divertikel kann vollkommen dem der Appendixschleimhaut gleichen. Lieberkühnsche Drusen sind oft aufindbar, seltener allerdings Lymphfollikelbildung. Das Epithel hat oft seine zylindrische Form beibehalten. es kann aber auch niedriger, kubisch werden, ein Befund, der als Regenerationstorgang der Schleimhaut nach Entzündungsprozessen aufgefaßt wird (r. Brunn, Simon, O bern dorfer) und nicht als Folge einer Schleimhautuberdehnung anzusehen ist oder als Folge gesteigerten Innendrucks in der Appen- 
dix, findet man doch diese Epithelabplattungen nur in den Divertikeln, dagegen in den zugehörigen Appendices nicht, in ihnen vielmehr wohlerhaltenes Zylinderepithel, obwohl in dem Lumen der Appendices doch auch übernormaler Druck besteht. L 'brigens findet man ja kubisches Epithel ebenfalls bei Schleimhautregenerationen bei ulzerosen Darmschleimhautprozessen, analogen „Heilungsvorgängen" der Schleimhaut wie bei der Divertikelbildung. Oft ist aber das Divertikellumen nicht vollig mit Lpithel ausgekleidet, was bei besonders groben Schleimhautdefekten nach Appendicitis perforativa der Fall sein soll. Die Wand der Divertikel an solchen epthellosen Stellen ist bindegewebiger Natur (Oberndorfer). Man sieht also, dab je nach der Genese und der (iroße der durch dic roraufgegangene Appendiritis entstandenen Muskeldefekte in der Appendix eimmal die (irobe der Divertikel selbst sehr variert, daß auch dic Epithelauskleidung der Divertikel normalen (harakter tragen kann, andererseits , alle Stadien der Epithelregeneration" (O) berndorfer) zu beobachten sind.

Das pathologisch-anatomische Bild der eimzelnen Divertikel kann also sehr verschieden sem. (ianz besonders bemerkenswert erscheint mir bei der Pathogenese der entzündlichen Wurmfortsatzdivertikel, dab zwe Schichten der Appendixwand Infektionen gegenuber besonder widerstandsfähig zu sein scheinen, das ist cinmal die Serosa (A $-c h$ off), dann aber auch die Mucosa. Schenkt man gerade diesen Punkt ein wenig Aufmerksamkeit, so wird man $a b$ und zu anf Fintzundunssbilder stoben, wo inmitten von Granulationsgewebe Binder von wohlerhaltenem Schleimhautepithel gefunden werden, welche keine Legencrationszeichen crkennen lassen, bei denen in Gegenteil reichliche Schleimmassen in der Umgebung Zeugnis fur ihre lebhafte physiologische Tätigkeit ablegen. Auffallend ist, wie lange solche aus ihrem Zusammenhang gerissene Epithclien oft zu langen Zellbandern vereingt, ohne sichtbare Degenerationserscheinungen persistieren können. Unsere Fig. I zeigt ein solches Bild. Besundere Aufmerksimkeit hat diesen Epithelzellbändern Oberndorfer zugewandt. Lr fand sie auch außerhalb der Appendix in Schleimmassen eingebettet, die ihre Entsteliung geplatzten Divertikeln verdankten.

Da bei den von Oberndorfer beschriebenen Fällen due 
Appendixoperationen schon „lange Jahre" zurücklagen, die Eplthelien sich also in fremdem Boden gut implantiert und erhalten hatten, ohne eine wesentliche Vermehrung erfahren zu haben", so ist damit der Beweis erbracht, daß tatsächlich außerhalb der Appendix Schleimhautepithel implantationstähig ist und in seiner Tätigkeit der Schleimproduktion fortfahren kann. Diese wichtige Feststellung $\mathrm{Oberndorfers}$ hat bisher keine Widerlegung erfahren. Damit kommen wir zu der weiteren Frage: Was wird a us geplatztem Appendixdivertikel, was aus dem in die lBauchhöhle ergossenen Schleim, vor allem

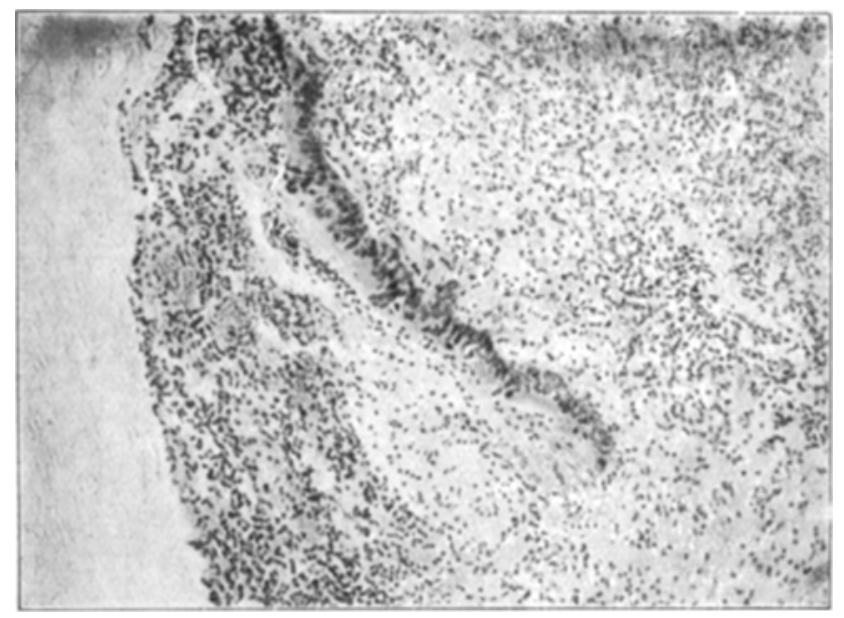

Fig. I.

Lose Zellbànder inmitten von Granulationsgewebe bei Appendicitis.

was aus den ihn produzierenden, unter Umständen mit in die freie Bauchhöhle rerlagerten Schleimhatepithelien der Appendixmucosa?

Während hinsichtlich der pathologischen Anatomie der Wurmfortsatzdivertikel und auch ihrer Genese kaum noch Zweifel auftauchen können, so ist doch hinsichtlich der Pathologie der Folgezustände des geplatzten Wurmfortsatzdivertikels keineswegs jede Fragestellung beantwortet. Mangels eindeutiger Namengebung dieser Folgezustände eines geplatzten Appendixdivertikels spricht man in Anlehnung an das ähnliche Krankheitsbild der Pseudo- 
myxoma pernonei als Folge emen geplatelen Ovarialkystomes schlechthin von Pseudomyxomil ex processu vermiforme.

Die Zahl der beschricbenen falle in nicht grolis, ieder in dieser Frage gebrachte lietrag bezinlich obiger Fragentellung erwünscht. E. Ir raenkel verfügt wohl uber die aungedehnteste Kemntnis dieses Krankheitsbildes, aut das er 1 yo I als erster aufmerksam gemacht hat. Secine Untersuchungen galten zunächst der Frage, ob es sch bei der Schleimbildung in der freien Bauchhöhle ron der Appendix herrührend um einen glewhartıgen pathologischanatomischen Vorgan: handelt, wie bei dem Pscudomyxoma peritonel, herruhrend ron einem seplatzten ()varialkyston. Insbeson dere galt es Fraenkel zu klären, ob die Schlembildung ex processu vermiformi allem von dem obliterierten W'urmfortsatz ihren Ausgang nimme, oder ob Schleimhatutepithel der Appendix auf dem I'eritoneum implantationsfähig ist und das Implantat die Fähigkeit besitzt, weiter Schleim zu produziercn. Diece Pragestellung hat eine besondere Bedeutung. Werth, der Entdecker des Pseudomyxoma peritonei als Folge emes geplataten Ovarialkystomes, hat swar in den im Mbdomen gefundenen schleimgefullten $Z_{y}$ sten niem:1 1 Epithclienausbildung gefunden, die Zystenbildung also als emen (roganiationuprozel's seiten des l'eritoneums autgefaßt; dagegen gelang Ohlshatsen, I't annenstiel. Polano und nicht zum wenigsten E. Fratenkel selbst einwandfrè der Nachwers epithelialer Zellausbildung der peritonealen Schleimzyaten. Hierdur h ist dem bilde der Pseudomyxoma peritonei als Fulge eines geplitaten Ovarialkystome durch seine Implantations-Metantasenbildung der Stempel des malignen Tumors aufgedrückt. In der 'Tat ist ja auch der klinische Verlauf dieses Krankheitsbildes in den weitaus mesten liallen der des malignen Tumors. Unter den Einchenungen der Kishexie kommen die Tráger dieses Leidens fast alle zu Tude.

Nur wenige Fälle ron IJeilung sind bexhrieben, so ron Hirth, Amann, Toth, Gottschalk und Pannenstiel. Dicser Ausgang, der allerdings eine seltene Aunnahme darstellt, wird ron E. Fraenkel überzeugend so erklärt, dals an vielen Stellen der Haupttunor und in einem groben Teil der als Implantationsmetastasen aufzufassenden Bildungen, selbstverständlich das 
ausschcidende Epithel zugrunde geht. Damit ist dann die Quelle für die Bildung neuer Pseudomyxommassen ausgeschaltet. Pfannenstiel führt die Zerstörung der Epithelien auf die Peritonitis zuruck. Jedoch, muB in allen Fällen die Prognose ernst gestellt und der früher oder spater erfolgende Tod an Kachexie als die Regel angesehen werden“. Auch wir verfügen über einen Fall (Privatsammlung Professor Konjetzny), bei dem das Leiden zum Tode geführt hatte. Nikroskopisch fand sich in den missenhaften Zysten überall eine Zylinderepithelauskleidung der Zystenwandungen.

Steht mithin das klinische Bild des Pseudomyxoma peritonei nach geplatztem Ovarialkystom ebenso wie das pathologisch-anatomische wohl absolut cindeutig und allerseits wohl anerkannt vor uns, so hat bei dem Pseudomyxoma peritonei ex processu vermiforme weder das klinische noch das anatomisch-histologische IBild in allen Fallen ein immer einheltliches (iepräge.

Zwar stellt igor E. Fraenkel an einem Falle fest, daß im Gegensatz zu dem Pseudomyxoma nach geplatztem Ovarialkystom das Pseudomyxoma ex processu vermtforme klinisch einen benignen Verlaut nimmt und die Schleimbildung bei dresem Krankheitsbild nicht von Implantationsmelastasen verlagerten Appendixschleim hautepithels herrührt. Auch sind seitdem zweifellos eine Relhe einschlagiger Fälle von Neumann, L, eontjew, Placzek. Honneker und Trotter eingehend beschrieben, die sowohl in klinischer als vor allem pathologisch-histologischer Beziehung vollkommen den Fracnkelschen Beobachtungen gleichen. Doch sind andererseits einige Fäle veroffentlicht, die cine Abweichung sowohl in histologischer als auch klinischer Bezichung ron den Fraenkelschen Fällen bieten, deren Deutung mit der von fir a en $\mathrm{k}$ el gegebenen nicht vereinbar ist.

Es steht demnach zur Entscheidung, ob bei dem „Pseudomyxoma peritonei“ die Schleimbildung allein aus dem geplatzten Appendixdivertikel herrührt; man mithin also die Bezeichnung Pseudomyxoma peritonei besser fallen läßt, etwa zugunsten , Ruptur eines zystisch entarteten Proc. vermiformis und konsekutive . Insammlung von gallertigen Schleimmassen in der Bauchhöhle (Fraenkel)“", oder obdieschleimbildungronder Täigkeit ver- 
lagerter normaler Schleimhautzellen der Appendixm ucosa herrührt bzw. herrühren kann, analog den Folgezuständen nach geplatztem Ovarialkystom.

Sehr wichtige einschlägige Veroffentlichungen über diese Frage sind bis 1912 erschienen und von E. Fraenkel selbst kritisch bewertet. Die Falle von Trotter, Honneker, Neu$\mathrm{mann}$ usw. finden in ihrer Deutung $\mathrm{Fraenkels}$ Anerkennung. Als nicht absolut eindeutig erscheinen ihm aber die Fälle ron H ü ter und M e r kel zu sein - wir werden weiter unten zu diesen Fällen noch Stellung nehmen - hat doch $H$ uter leststellen müssen, daß3 der Verschluß des proximalen Appendixlumen durch eir Karzinom herbeigeführt worden war, ,eine bedenkliche Komplikation, die den Wert der an sich lehrreichen Mitteilung für die Frage der Pseudomyxome beeinträchtigt". Auf der Pathologentagung in Erlangen I 9 Io habe auch $S \mathrm{ch}$ morl beziglich des Merkelschen Falles Bedenken getragen, ob es ich nicht etwà um ein Carcinoma muciparum handeln könne. Den Fall, bei dem Oberndorfer einwandfrei auf der Appendixserosa implantiertes Schleimhautepithel der Appendix fand, das sich seiner Ansicht nach lange Zeit funktionsfähig erhalten hatte, deutet Fraenkel so, daß die physiologischen Schleimhautzylinderepithelien sich allenfalls, aber keineswegs regelmälsig, in unmittelbarer Umgebung des Organs, aus dem sie stammen, anzusiedeln, dort auch längere Zeit ev. noch $z u$ funktionieren vermogen, ohne aber ,eine wesentliche Vermehrung zu erfahren“.

Daß die Befunde Oberndorfers in ihrer Deutung von Fraenkel Anerkennung gefunden haben, scheint mir von Wichtigkeit zu sein. Es erübrigt sich, an die nähere Beschreibung der Fälle von Neumann, Honneker und Trotter zu gehen, da sie vollkommen das von Fraenkel aufgestellte Postulat erfüllen. Ein eigener Fall möge zur Erläuterng des Fraenkelschen Standpunktes dienen:

J.-Nr. I81o 192I. W. K., 3I J. Feinmechaniker, komm mit starken Leibschmerzen und Brechreiz in die Klinik. Hat fruher manchmal Leibschmerzen. I918 eine schwere Blinddarmenzzindung gehabt. Seit dieser Zeit mehrere kleinere Blinddarmentzundungsantalle.

Junger kraf:iger Mann in gutem Ernihrungszustand mit gesun den inneren Organen. Bauch $r$ unten, aber auch l. unten dructi- 
cmpfindlich und gespannt Gegend vor Magen und Leber schmerzfrei Rektaluntersuchung: Schmerchafter Douglas. Temp. 38,9 Grad.

Sofortige Operation in Athernarkose (Verdacht beginnender Pcritonitis ex appendicatide!: Nach Eroifnung der Bauchhohle quellen gallertartige ubelrıechende Massen hervor. In Gegend des Co cums große Nengen dieser Schleimmassen Appendix stark verwachsen und in den sie bedeckenden Srhleimmassen schwer auffindbar Typische Appendektome. Dranage der Bauchhohle. Nach anfanglicher Eiterung Heilung o. B.

Práparat (F1g.2) Langsschnitl durch de Appendix zeigt diese proximal obliceriert, distal zystisch in Divertikelform erweitert Dicses ist an der dem Mesenteriolum abgekehrten Seite perforiert. Schleimhaltiges, ziemlich derbes weiBirches Gewebe ats der $\mathrm{Tm}$

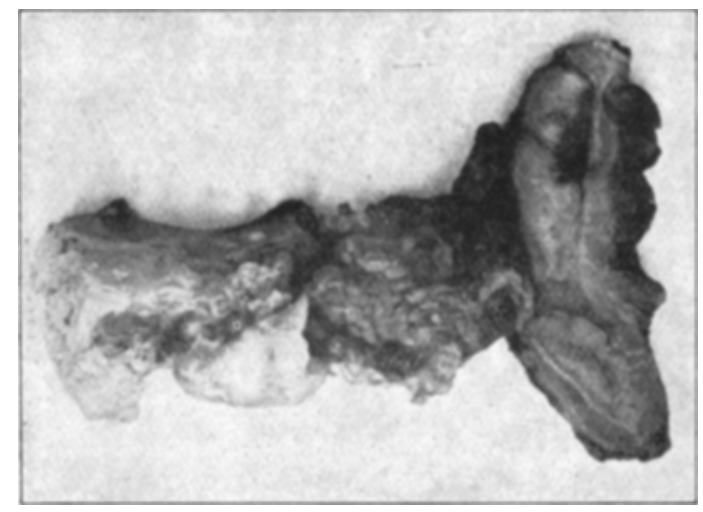

Fig 2

gebung der Appendix laßt makroskopisch hinsiclitlich seiner Struktur nuhis Vaheres erkennen

Mikroskop. Befund Proximales Ende der Appendix schwielig verändert. Lumen auf etwa $\mathrm{I} \mathrm{cm}$ Lange vollkommen durch Narbengewebe verschlosen Schleimhaut bis auf kleine Inselchen noch vorhandener Drusenschläuche geschwunden. Distal beim Übergang der obliterierten Appendixpartie in den zystisch erweiterten Teil in der Muscularis der Appendix reichliche kleinzellige Infiltration, Gewebsnekrose und Blutungen. Die Gefaße des Mesenteriolums sind prall gefullt. In ihrer Umgebung kleinzellige Infiltration. An der dem Mesenteriolum abgckehrten Seite ist die Appendixwand stark narbig verandert und schlagt sich etwa $\mathrm{I} \mathrm{cm}$ unterhalb der Obliterationsstelle der Appendx in einem $\mathrm{I} \mathrm{cm}$ breiten Defekt nach außen um und verliert sich dann in dem außen der Appendix aufsitzenden Bindegewche Dic Schleimhaut ist in lebhafter 
Wucherung unterhalb des zystisch erweiterten Teil der Appendix. Sie bildet bizarre papillomatöse Bilder, bald reich verästelte Verzweigungen mit hohem Zy'indercpithel-Besatz, bald d.chtstehende tast $1 \mathrm{~mm}$ lange Zapfen mit dem gleichen Epithelbesatz. Besonders in der Richtung der Perforationsstelle hin folgen diese Schleimhautzapfen dem Zug ihrer Unterlage, folgen gewissermaßen der Stromrichtung nach außen, schlagen sich auch auf ganz kurze Strecke aut die umgekrempelte Muscularis um. Sie horen aber in allen Schnitten dann in der Muscularis auf, obwohl ihre narbige Unterlage sich gleıchmäßig in das außen die Appendix umgebende Bindegewebe fortsetzt. Dieses Bindegewebe bildet kleinere und groBere Zysten, deren Wandung aber nur aus Bindegewebszellen besteht. In den Zysten in scholligen Häufchen hier und da Schlcim von feinen Bindegewebszugen umsponnen. Nur wenig kleinzellige Infiltration. Nirgends ein Vorhandensein von an Mucosaepithel erinnernden Zellverbainden. Der gleiche Befund wird festgestellt bei einem Schleimstückchen, das aus der Umgebung der Appendix exstirpiert wurde.

Diagnose. Appendicitis, älteres geplatztes Appendixdivertikel mit Schleimproduktion in die Bauchhöhle. Alterer bindegewebiger Organisationsprozeß des Schleimes Quelle der Schleimbildung lediglich die Divertikelschlcimhaut.

Komplizerter liegen die Verhältnisse bei dem Hüterschen und Merkelschen Fall. Die an ihnen erörterten Fragestellungen sind so wichtig, daß wir die Fälle kurz skizzieren müssen.

H üterscher Fall: $25 \mathrm{j}$. Mann wird wegen doppelscitigen Leistenbruchs operiert. Bruchsackinhalt zusammengeknuilltes Netz, körnerartige Zystchen enthaltend. - I4 T. post op. Exitus an Lungenembolie Peritoneum und der ubrige Teil des Netzes zeigen, ausgenommen die Ileocöcalgegend, normales Aussehen. Appendix und anschlieBender Teil des Cöcums von feinen Membranen eingehullt, die großere und kleinerc schleimgefüllte Zysten enthalten Distales Appendixende verdickt, an der Spitze eine dünnwandige Prominenz, Schleim enthaltend Außen der Prominenz aufsitzend kleine schleimhaltige Zysten. Proximaler Teil der Appendix durch einen Tumor verschlossen.

Mikroskop. B ef und: Obturierender Tumor; Karzinom mit gewahrtem Drüsentypus.

Im Lumen der Appendix Scbleim; nahe der verdinnten Wandpartie im Lumen ein gefaltetes abgelöstes Band von Schleimvakuolen enthaltenden Deckzcllen. Dieses einschichtige, viele Becherzellen enthaltende Zellband verläuft ganz gradlinig, oft ist es abgelöst, halskrausenartig gefaltet, als ob der Umfang der Unterlage sich verkleinert hätte. Bei Annähcrung an die dünne Wandpartie werden die 
Zellen immer klemer, Schwund der Becherzellenbildung. Die Zellen werden ganz atrophisch, platten sich schlieBlich ganz ab, um endlich an der dunnsten Wandpartie vollig zu fehlen. An der dunnsten Parlie Defekt in der Appendixmuskulatur. Die der Appendix aufsitzenden Zysten kommunizieren vielfach miteinander und haben durchweg bindegewebige Membranen Ihr Schleiminhalt enthält beigemischt neben polynukleären Leukocyten, Lymphocyten, Bindegewebszellen und Fasern eigentumliche Zellen, die in mehrfacher Schicht in Bändern bis zu 20 und mehr Zellen nebeneinander liegen. oft in drusenartiger Konfiguration. Eine Basalmembran fehlt Inn rhalb des gleichen Zellbandes wechselt der Zelltypus von hochzylindrischen bis zu kubischen, ja flachen Zellen. An den kubischen Zellen sogar Harchensäume sichtbar Derartige Zellverbände auch weitab vom Cocum und Appendix auffindbar. Auch riesenzellenartige Gebilde werden gefunden. Dic bindegewebigen Hüllen der Zysten sind mi! mehrfacher Schicht platter Zellen uberzogen.

Merkelscher Fall 79 j. Mann, an Pneumonie zum Extus kommend. Behandlung wegen Prostatahypertrophie, Cystitis usw. In der Anamnese keine Appendicitis.

A ut opsie: Der ganze Bauch voller gallertiger Massen, die auch an Leber und Milz adhärieren. Mikroskopischer Befund wie bei dem Fraenkel-, Hüter- und Neumannschen Fall usw. Nur an der Milzkonkavitat finden sich große, miteinander z. T. konfluierende, von Schleim erfullte Zystengebilde einwärts nach dem Hilus zu gelegen. Wahrend sonst der endothelberaubten Milzkapsel die Schleimmassen direkt aufgelagert sind, zeigt sich im Bereich der Zysten ein regulärer Epithelbelag, und zwar findet sich die Epithelauskleidung mitunter kubisch, dann in immer höher werdendes Zylinderepithel ubergehend Dieses Zylindercpithel zeigt ausgesprochene Schleimbildung in Form von Becherzellenbildung, bald treten die Epithelformationen in Form von Papillen, ja schließlich sogar drüsenartigen Gcbilden auf, so daß tatsaichlich besonders auf Flachschnitten Drïsenlumina aus Becherzellen bestehend zu konstatieren sind.

In jungster Zeit hat sich zu diesen „im strikten Gegensatz zu den Untersuchungen E. Frae nkels" stehenden Beschreibungen noch ein dritter gesellt, der Fall Comolles.

$6 \nmid \mathrm{J}$. Mann wegen fistelnden Nabelbruchs operiert. Bei Eroffnung des Peritoneums Mengen truber, gallertiger Flüssiglkeit. Peritoneum diffus besetzt mit myxomatösen Massen. Exitus.

A u topsie: Peritoneum parietale, Leber, Milz, Darm und Netz bedeckt mit gallertigen Massen, z. T. in Zysten mit der Unterlage fest verwachsen.

Wurmfortsatz in dicke Gallertmassen eingelagert, in seinem proximalen Drittel unverändert. Darauf obliterierter, ca $3 \mathrm{~cm}$ lan- 
ger Strang, mit zystuscher Erwelterung an seinem distalen Ende. Zyste selbst lecr, enthalt kleine Purforation in weitere schleimgefullte Zyste, die ihrerseits wieder inmitten gleichartiger Zysten liegt, die schleimgefullt den Wurmfortsat $\mathrm{d}_{\text {icht }}$ umgeben.

Mikroskop B of und: Deckzellenausk'eidung fehlt sowohl an Innenwand der distalen Appendixerwelterung wie mereich des Überganges der Appendix in die Zyste. Wandungen der der Appendix außen autleyenden Zysicn in der Hauptsache aus neugebildetem Bindegewebe bestehend Die Innenwand der Zyste, wchche mit dem Appendixlumen kommuniziert, zeigt an der dor Kommunikationsöfnung zunächst gelegenen Stellc cinen platten, einschichtigen Zellbelag, der allmahlich hoher wird und kubische Gestalt annimmt. Dann Strccken teils mit, teils ohne Deckzcllenbelag Wandteile der gleichen Zyste z. T mit hohen zylundrischen Deckzollen ausgekleidet, vereinzelt Becherzcllenbuldung aufweisend. Dic ubrigen in der Nähe befindlichen Zysten mit Zylinderepithelaunkleidung und oft deutlicher Bccherzellonbildung

Net\%- und Maschenräume mit homogenen Schleimmassen angefullt, teilwerse mit Zelibesat\% von kubischem bis hochzylindrischem Zelltypus, of zahlreiche Becherzellen enthaltend Nirgends Mehrschichtigkeit.

Das gleiche Bild an der Milzoberfliche, an Ixber und parictalem Peritoncum. I'berall, wo Schleimmassen dem Poritoneum direkt auf liegen, ist die Deckzellenschicht zugrunde gegangen Dic subserose Schicht zeigt vielfach starke Rundzellenintiltration

Diesen drei Fallen reiht sich norh ein vierter Fall von E. Michaelson an, eine Arbeit, die mir nur im Referat zugänglich war.

$46 j$ Mann, bei dem zufällig gelegentlıch der Autopsie ene proximal oblitcrierte Appendix gefunden wurde, whingesen das distale Ende in einen walnuBgroßen schleimhaltigen Klumpen umgewandelt war mit deutlicher dreickiger Perforation Das Netz war ebenfalls von Schleimklumpen besctzt, dic von M. als Implantationsmetastasen von Appendixschleimhatutepithel angesprochen werden Leider ist au, dem Referat nicht rsichtlich, ob diese Ansicht Michaelsons mikroskopisch erhartet ist Der Fall ist also fur uns kritisch nicht verwendbar

Wic schwierig die Kritı der Fälle von Hüter, Merkel, Comolles ist, zeigt die Beurteilung, die Hüters und Mer. kels Fall auf den Pathologentagungen erfahren haben. Wir erwähnten ja oben schon, daß der Merkelsche Fall io Io allein von Schmorl im Hinblick auf den unter Schmorl bearbeiteten Honeckerschen Fall möglicherweise als Carcinoma muciparum an- 
gesprochen wurde. Andererseits wurde auch von ihm die regelmäßige Zylinderzellenbildung in den Präparaten im Gegensatz zu den Epithelveränderungen bei den schleimbildenden Karzinomen ausdrücklich hervorgehoben. Hingegen setzten $\mathrm{Hauser}$ und Nopurgo sich restlos für die Merkelsche Deutung der vorgelegten Präparate ein. Unter Bezugnahme auf die Schmorlschen Einwände hält E. F ra e n kel sich auch für berechtigt, ,nur solche Falle zur Beurteilung der Frage der Pseudomyxome peritonei ex processti vermiforme heranzuziehen, bei denen an keinem der in Betracht kommenden Unterleibsorgane irgendeine auch nur auf Krebs verdächtige Neubildung besteht oder bestanden hat". Wenn Fraenkel aus diesen Gründen den Hüterschen Fall ablehnt in seiner Deutung als l'seudomyxoma peritonei ex processu vermiforme, so wird er wohl wenig Widerspruch finden, während der Merkelsche Fall, durch vielseitiges autoritatives Urteil gestützt, den ihm in seiner Erklärung zugewiesenen Platz behauptet hat.

Was nun den Comolleschen Fall betrifft, so int auch dieser in manchen Punkten im Fraenkelschen Sinne nicht „einwandfrei“, was übrigens von Com olle selbst zugegeben wird. Pathologischanatomisch unterscheidet sich dieser Fall kaum ron dem Merkelschen Fall, nach dem ganzen pathologisch-anatomischen Befund besteht für die Annahme des Ausgangs des ganzen Prozesses von einem moglicherweise vorhandenen, aber nicht mehr nachweisbaren Karzinom der Blinddarmspitze wohl kaum ein Anhaltspunkt, ebensowenig für die Annahme eines Gallertkarzinoms auf der Basis abgeschnurten Darmepithels oder sonstiger Fehlbildungen. Völlig auszuschließen ist aber natürlich diese Möglichkeit nicht. Gegen die Diagnose Carcinoma gelatinosum scheint Comolle einmal zu sprechen. daß atypische Zellwucherungen, mehr oder weniger kompakte Zellhaufen nicht gefunden wurden, nirgends auch kolloide Degeneration der Zellen konstatiert werden konnte, der ganze Prozeß in Gegensatz zu dem destruktiven Wachstum eines Karzinoms ,,rein produktiven Charakter" mit starker Bindegewebsentwicklung trug. Würde man an ein ron dem Peritonealepithel (Fndothel) ausgehendes Karzinom denken, so wurde besondere Schwierigkeit die Deutung der nachgewiesenen Zylinderzellen, besonders aber die der Becherzellenbildung machen, da der Nach. weis, daß Metaplasic von Peritonealepithel in Schleim produ- 
zierendem Zylinderepithel rorkommt b $<w$. möglich int, experimentell bisher nicht erloracht werden konnte.

Allerdings ist es $\mathrm{L}$ ubars $\mathrm{ch}$ gelungen, ber cntzundlichen Pro zessen seroser Haute die I'mwandiung von platten Scrosadeckzellen in kubssche, ja zylindrische Zellen nar-hzuweisen, in Fallen von Endocarditis sogar an Becherzellen erinnernde Zylinderepithclformen auf den Herzklappenhervorragungen zu finden

Man kann alsu nach den liefunden L u ba r sch s prinzipiell dic Miglichkeit einer Metaplasie von Peritonealepithel in zylindrisches, ja becherzellenhaltiges Epithel nicht in Abrede stellen. Aber, wie gesagt, sind derartige Befunde, der Nachweis der Umwandlung ron I'eritonealepithel in Zylinder-oder gar Becherzellenepithel mit Schleimbildung in den experimentellen . Irbeiten uber die Entzundungen in serosen Hohlen nicht erbracht.

In den bekannten Versuchen Mönckeberg mit Lykopodiumsporenstaul, Entzundung in serosen Hohlen stelle dieser Auten als erste Phase cine Schadrgung der Decksellen fest und als zweiten Abschnitt went uber de Grenzen des geschadigten Bezirkes hinaus reichende progressive Vorand rungen des Deckejuthels (Endothels). bestehend in emer Formanderung des Endothels in kubischen, ja in zuckerhuttormiges und Zylinderepithel.

Die Herzogschen Untersuchungen gleicher Art uber die Wirkungr ron ausgeleerten Fettmisscn aus einem greplatzten OvarialDermoideystom auf das Peritoneum ahneln 10 ihrem Ergebnis denen Monckebergs insofern, als von II croog ebenfalls an einigen Stellen das Zugrundegehen von 1)eckzellen fostgcotellt wurde, spaterhin auch wh thm als Regeneration ron Serosideckzollen ange sprochene Prozesse beobachtet wurden, whei deutleh kubsor he Zellen sich herangebildet hatten, hingsesen aber keme Zylinderzellformen boobachtet wurden

Im Gegensatz hierzu stehen die hier nochmal, zttierten Merkelschen Befunde, der zwar auch bei einem seiner Fälle in der Tiefe des Beckens unter Schleim wohlerhaltenes Epithel fand, das, wie bei den Mönckebergschen Versuchen, kubische Form angenommen hatte, aber andererseits implantiertes hohes Zylinderepithel fand, das mit den oben beschricbenen kubischen Zellen nicht verwechselt werden konnte, sich von dem Peritoncalepithel, wo es noch vorhanden war, immer scharf unterschicd, zahlreiche Becherzellenbildung aufwies und Neigung hatte, sich flachenartig auszubreiten und papillenartige Vorsprünge zu bilden, ja Drusenform anzunehmen. 
Leider entbehrt der Comolle sche Fall auch der Übergänge von Appendixschleimhautepithel in hohes, den Zystenraum auskleidendes Zylinderepithel, ein Mangel, der die Beurteilung des Falles sehr erschwert. Immerhın aber gleichen die in den Zysten gefundenen Zellatiskleidungen so den von $M$ erkel beschriebenen Zellen, daß eine Identifizierung mut diesen seitens $\mathrm{Com}$ olles nicht unberechtigt erscheint, obwohl sichere Beweisfuhrung nicht erbracht werden kann und Irrtumsmöglichkeiten zugegeben werden mussen.

Der Comollesche Fall ist also aller Wahrscheinlichkeit nach dem Merkelschen ähnlich, wenn nicht ihm wesengleich.

Während Merkel, Oberndorfer und Comolle die ron ihnen beschriebenen Zylinderepithelfornen mit Becherzellenbildung als versprengtes und dann implantiertes Darmschleimhautepithel ansehen, sucht $H$ üter die von ihm gefundenen Zellen hohen Zylinderepithels, ja sogar die Becherzellbildung als eingewanderte Peritonealdeckzellen zu erklären. Seiner Ansicht nach liegt kein Grund zur Annahme vor, dab a us den verhälth is mäbig niedrigen Schleimhautepithelien des Appendix bei ev. Implantation auf das Perıtoneum hohe zylindrische Formen hervorgehen. Als Stütze fur seine Behauptung dienen ihm gefundene Zellbänder, in deren Verlauf zylindrische, kubische und niedrige, fast platte Zellen nebeneinander gefunden wurden.

Unserer Ansicht nach sind $\mathrm{H}$ üters Folgerungen unrichtig, dil wir im folgenden zu beweisen gedenken, daß einwandfreies Appendixschleimhautepithel aus normaler Konfiguration in hohes, fast durchweg aus Zylinderbecherzellen bestehendes, palisadenartig geordnetes Epithel übergehen kann. Andererseits geht ja aus den Beschretbungen über die Appendixdivertikel einwandfrei hervor, daB besonders bei Überkleidung von Defekten Darmschleimhautepithel in niedrige Epithelformen übergehen kann, worauf wir ja eingangs schon zu sprechen kamen.

Es sei nun gestattet, auch zu dieser Frage einen eigenen Beitrag zu liefern.

Frau $\mathrm{I}^{\circ}$, cinc $78 \mathrm{j}$. Frau, kommt in de Klinik zur Leistenbruchoperation. Der Lestenbruch $\mathrm{r}$. soll seit reichlich $40 \mathrm{~J}$. bestchen und in letzter Zeit gewachsen sein. Durch seine Größe wird er lästig. Vor gut $30 \mathrm{~J}$. ist der Leistenbruch schon enmal inzidiert worden, wobei sich , whserige Flussigkeit" entleert haben soll Eine Blind- 
darmentzundung hat Pat. weder in letzter Zeit noch in fruheren Jahren durchgemach:

Alte, aber gesund aussehende Frau mit gesunden inneren $\mathrm{Or}$ ganen. In $r$ Leistenbenge ein gut zweifaustgroßer, derber Tumor, der ziemlich prall ist und keine Fluktuation zeigt. Er ist beim Betasten nicht schmerzhaft

Diagnose: Hernia permagna irreponibilis

Operation in Lokalanasthesie: Schnitt uber die Hohe des Tumors in Verlaufsrichtung des Leistenkanals. Nach Durchtrennung der Haut kommt man gleich in eine Höhle, aus der dicker Massen zahen, festen, tischlerleimfarbenen Schleims sich entleeren, der eine große Klebrigkeit aufweist. Die zahen Schleimmassen werden nun zum Teil ausgeraumt, zum Teil in der zweifaustgroßen Hohle belassen ${ }^{1}$ ). Es gelingt nun, unter Exzision eines Hautstuckes einen großen Le'stenbruchsack bis an den sehr engen L.iistenkanal zu isolieren. Er setat sich strangartig in etwa Bleistiftclicke in dem Leistenkanal fort. Nach Isolierung des Bruchsackes zcigt sich nun in seiner Tiefe die Appendix wic ein Birnenstiel in ihn einmundend. Sie ist in dem sehr engen, verwachsenen Leistenkanal bis auf einen stricknadeldunnen Strang obliteriert in einer Ausdehnung von etwa $3 \mathrm{~cm}$ Lange, um damn als auBerlich normale Appendix in die freie Bauchhohle ubersugehen Sie wird bis zu ihrer Eımunduug ins Cocum vertolgı und dort in typscher Weise abgetragen Bauchhohle völlig normal, desgleschen die gesichteten Darmteile. Kein Schleim im Abdomen. -- Tersorgung des Bruchsackes usw. Helung p. p.

Das gewonnene Praparat hat das Aussehen einer ricsigen Birne, wobei die Appendx von dem Bruchsackhals eng umschlosien dem Stiel der Birne entspriclut (siche Fig. 3 )

An den gehàteten Praparat (Fig 4 ) sicht man innen die Appendix mit deutlich sichtbaren Lumen einminden, um sich in einen tellerartigen, beinahe kreisrunden platten Ring fortzusetzen, der sich rings um die Appcndixeinmündung ausbrettet und glatte Oberfläche hat. Weiter distal ist die Bruchsackwand dunner, zeigt viele Zotten bildung und Vorsprunge, zwischen denen Schleim haftet, oder sammetartiges Aussehen, was durch die Buldung feinster Zöttchen bewirkt wird.

Mikroskopisches Bild: (uerschnit durch die Appendix unmittelbar bei ihrem Abgang aus dem Cöcum: In der Muscularis Narbenbldung sonst normale Konfiguration der Schlemhaut, Licberkuhnsche Krypten und Follikel An einer Stelle in der äuBeren

I) Die entleerten Massen wogen reschlich $\mathrm{r} \mathrm{kg}$. Die chemische I'ntersuchungr des Schlems durch den Chemiker Herrn Dr. Iagr r ergab Pseudomucin Der Schleim war steril. 
Muskellage rerlagertes Schleimhatepithel, daneben zystenartigus Lumen (Divertikelbildung?)

Querschnitt durch den obliterierten, strangformigen Teil dor

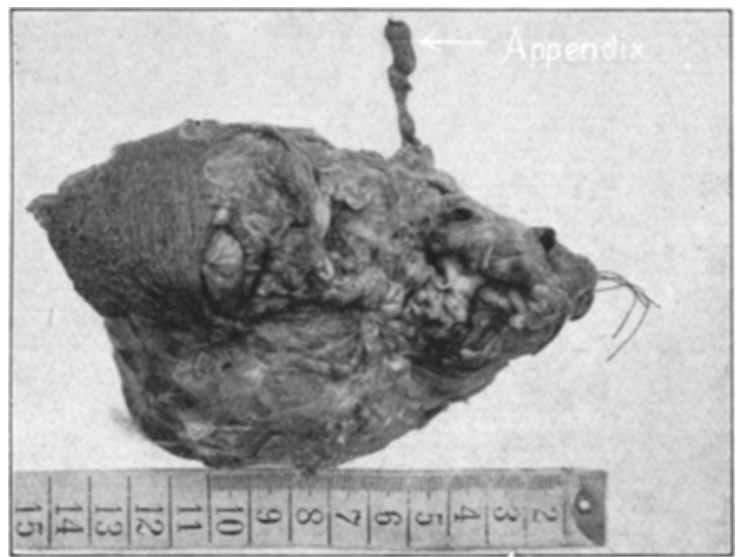

\section{Fig. 3}

Herausgeloster I3ruchsack, in den die Appendix hinemragt.

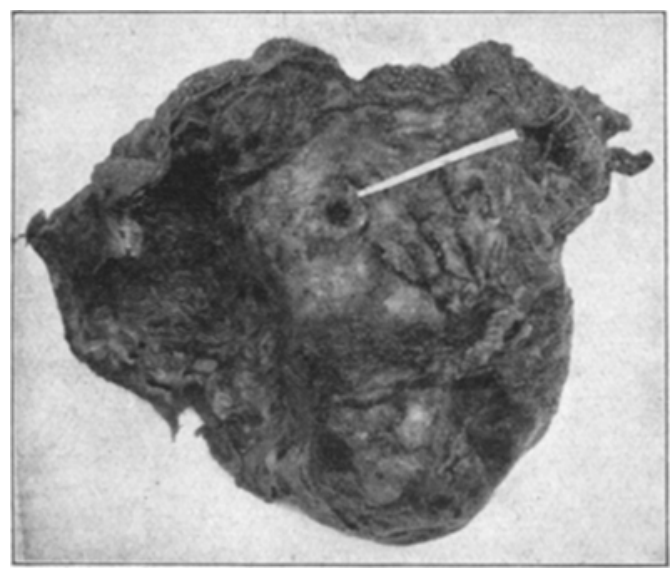

Fig. 4

Appendix: Nur Narbengewebe olne Infiltration. Daneben ziemlich starke, gut erhaltene, senkrecht zur Längsachse getroffene Gofaße. Schleimhautepithel oder Nuskulatur, sowie L_ymphfoilikelbildung ist nicht nachwejsbar 
Querschniti durch die Appendis unmutelbar von ihrer Einmundung in den Bruchsack (Firr 5): Bei gewahrter Zilichnung der Appendixwand starke Narbengewebe als Ersat\% der Muskulatur. Schleimhaut besteht aus halskrausenart:g angeordnetem hohem zylindrischem, beinalue ausschleeBiich Berherzellen enthaltenden Epithel in enschichtiger Lage, in engen Windungen gelegt, als sei die Unteriage zu kurz, k'me Kryptenbildung, keine Lymphfollikel. Die narbig verwandlelte Munculare mit der Bruchwand untrennbar verschmolzen zu emem hyalinen, fast kernlouen Bindegewebe. Langs.

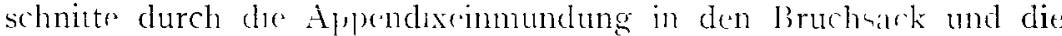
Umgelsung.

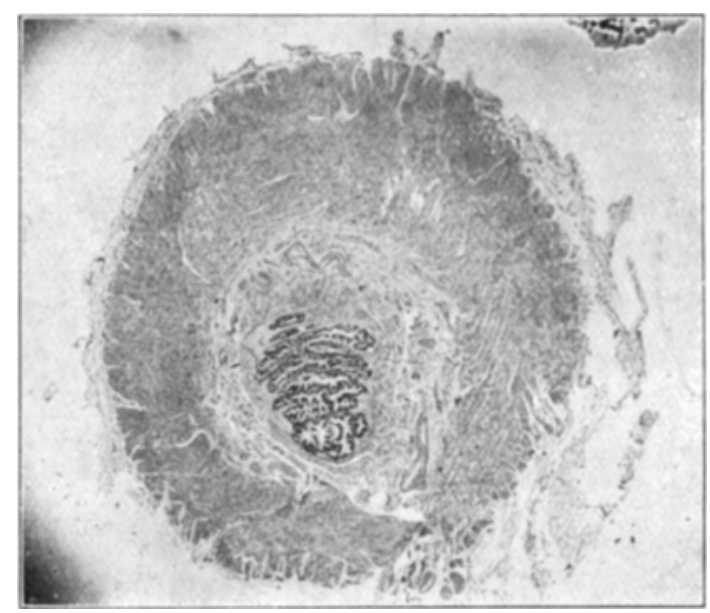

$$
\text { Iisg. } 5 \text {. }
$$

Appendix unmettclbar vor der Einmundung in den Bruchsack. Halskrausenartiges, hohen sylindrisches Becherıellenepthel. Fehlen der L,eber kuhnschen Drüscn.

Im Appendixlumen kurs vor der Eimmundung in dhe groibe Bruchsackhohle keine Schleimhaut feststelibar, digegen stcißt man sofort wieder aut Schleimhautepthel an der Emmundungsstelle der Appendix in den Bruchsack. Papillenarige Vorspringe ragen in dic Bruchsackhohlung hincm, dicht beset $t$ t mit hohem, durchweg aus Becherzellen bestchendem Epithel, das cinreihig sehr gleichmäBig einem dunnen, aus zartem Bindrgewebe aufgebauten, schr zahlreiche gut gefullte Gefäße rnthaltenden Stiel aufsıtzt, um dann der Bruchsackwand als I3elag entlangzulaufen, in einfacher Lage hochzylindrischen, rein aus Becherzellen bestehenden Epithels, ab und zu noch kleine Papillen bildend (Fig 6) 
Weiter peripherwärts an Stellen, bui denen auch makroskopisch gröbere Zottenbildung auffält, sind sehr wechselvolle Bilder. Phan-

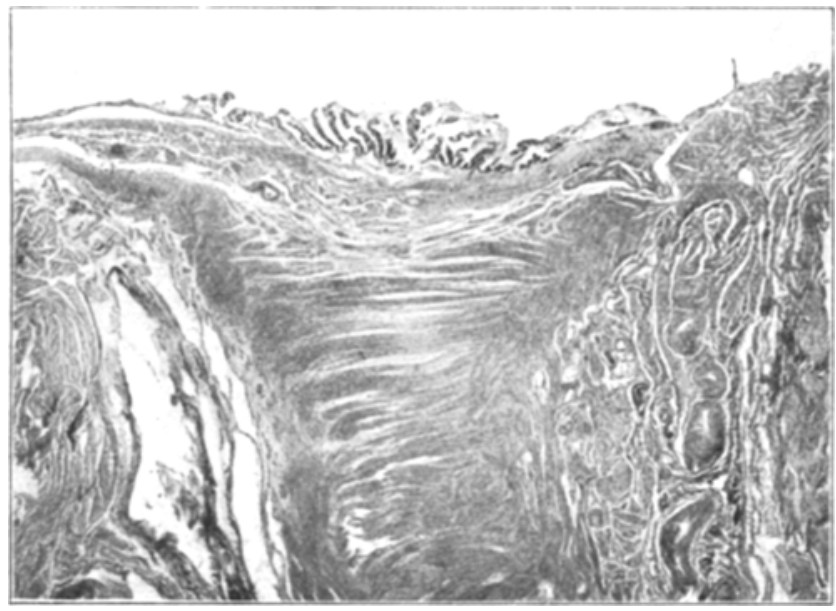

Fig. 6

Einmundung der Appendix in den Bruchsark

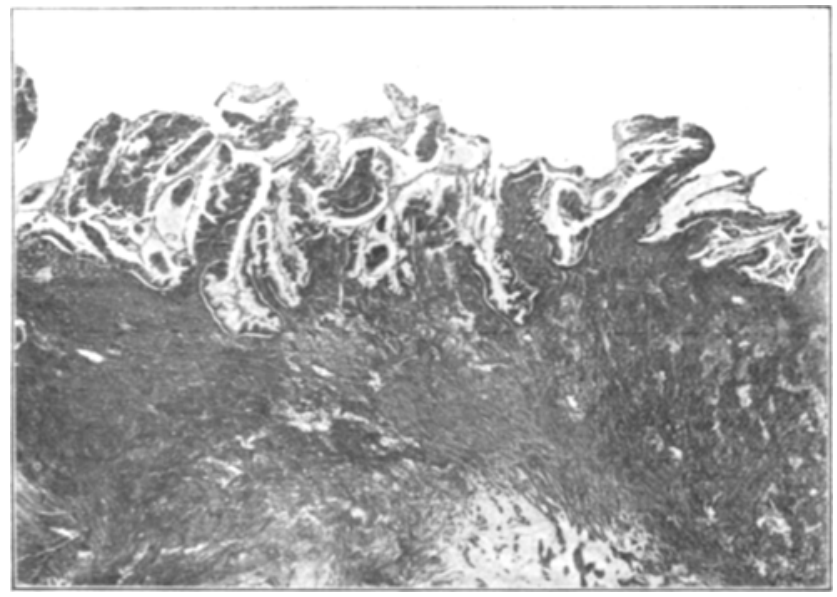

Fig. 7.

Papillomatose Wucher ungen im Bruchsack, hohes zylindrisches Becherzellenepithel.

tastisch geformte, selır reichlich große Gefaße haltende Bindegewebspapillen snd uberall ohne Unterbrechung von hohem, en- 
schichtıgen Zyhnderepithel, durchweg mit Becherzellenbildung, busetzt (Fig. \%). Cirobe, in das Bruchsacklumen hereinragende quer getroftene Papillen legen mit hoher Epithelkrause ringsum umgeben als Inseln im Schnit. Durchweg findet man nur allein diese beschriebene Epithelart Man kann das Epithel aut weite Strecken verfolgen, wie es den reichen Vald won Zotlen und Zittchen iber. klettert. Die Bruchsackwand baw. de von lhe morphologisch nicht zu trennende Wand der Appendis besteht aus breiten kernlusen Zugen hyalinen Bindegewrbes, das seh peripherwarts immer mehr aufsplitert, zwschen den cinsenen Zugen Sehlein emschlicßend. nach dem Bruchsacklumen zu vielfach mitenander verschlungenen, ,netzartigen" Charakter annehmend Zwinchen don Bindegewebsugen mehr oder "reniges CutäBbildung, her und da Kalkiblage rung, an wenigen Stellen sermgfugige, kleinedlige Intiltration, daneben große, blange, autgequollene Zellen, beinahe dremal 4 zroß als das chen beschriebene Zylinderepithel, deren Natur, ub) epithelialer IIrkunft oder mich, nicht festgestcll werden kann

Elwa wn dem großien queren Durchmesser des Bruchsackes beginnend. nach dintalwarts reschlir he zotten- and E'apllenbildung, die aber in ciegensatz 2 , den oben beschrebencn ohn Zylinderdeckzellenbelas sind 'Irot' mannigfach angelegtel Schnite' ge'lingt es niemals, in dem distalen Terl des Bruchnackes uberhaupt Epithelausklcidung testatstellen Eine scharfe Schridcgrena zuischen epithelbedeckten und cpthelfreien lapulen wirl nicht gefunden, ein Deckzellenbelag in dem distalen Teil des Bruchsackes wird uberhaupt vermißl. Bindegewelor, $z$ T. fein autgetasert, m Maschen Schloim sonschlebersd, rage in dir bchloimmanen mit reinem Gitterwerk hmein

Wemn wir das Camze zusammenfassen, so handelt es stch ein typisches I'seudomyxoma peritoni ex procesull remiforme. worüber nicht zu diskutieren ist. I'nser Fall hat mı dem Fraenkelschen gemeinsam das auberordentlich langame Wachstum, dan wahrscheinlich uber 30 Jahre gedauert hat. Die wasserige liussigkeit, die der Hausarzt ror reichlich 30 Jahren nach Inzision abgelassen hat, wird wohl auch Schleim gewesen sein, der rielleicht zum Teil noch nicht so eingedickt war wie späterhin. Die Benignitat des Prozesses paBt auch in den Rahmen des Fiankelachon l'ostulates 1 ).

Nach Entternumg der Appendix ist der I'rozels zum Stillstand gekommen, Metastasen sind nicht rorhanden. Cranz abgesehen von

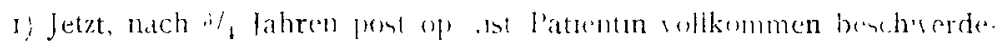
und rezidivfrei. 
der Seltenheit des kasuistischen Beitrags eines Pseudomyxoms in einem Bruchsack hat unser Fall gewissermaßen den Wert eines Experiments, das dartut, welcher Veränderungen ein verlagerter, abgeschnürter Appendixteil fähig ist. Insbesondere ist es die lirage nach dem Wachstum des Epithels, die interessiert. Fraglos isl, dâh in dem Bruchsack Epithclauskleidungen vorhanden sind, dic in kontinuierlichem Zusammenhang mit der aus der Appendixïffnung hervorgehenden Schleimhaut stehen und ausnahms. los nur diesen einen Zelltypus hohen Becherzellenepithels repräsentieren, das entweder in slatter Linie verläuft, stellenweise aber hier und da schon kleinste P'apillen bildet, oder andererseits den Besatz papillarer, oft bizarrer Wucherungen abgibt. Dieses Epithel unterscheidet sich in nichts ron dem halskrausenartig angeord neten Eptihel, das auf dem Appendixquerschnitt ummittelbar vor seiner Einmündung in den Bruchsack gefunden wird. Warum der Jeckzellenbelag hohen Zylmderepithels nicht in dem ganzen Bruchsack, besonders in seinen peripheren Teilen gefunden wird, ist nicht so leicht zu erklaren. Einmal konnte er bes der Operation durch Jupfen abgewischt rein, was (leshalb unwahrscheinlich ist. weil selbst in schleimbedeckten Teilen des periphersten Teiles des Bruchsackpräparates die Zylinderzellen sowie uberhaupt jeglicher Deckzellenbelag rermißt wurde. Eine andere wahrscheinlichere Deutung wäre die, daß die Wachstumsenergien der Appendixschleimhant nicht so weit reichen, die Epithelauskleidung bis in die periphersten Teile des Bruchsackes vorzutragen. Die in den peripheren Teilen des Bruchsackes gefundenen Bilder möchten wir vielmelr als Resorptions- bzw. Organisationsers heinungen deuten. we sie Fraenkel in seinen lällen beschreibt.

Inser Fall, notabene der einzige weibliche I'atient mil Pseudomyxoma peritonei ex processu vermiforme, hat nur in der italienischen Literatur einen Parallelfall, den ich zu zitieren deshalb nicht unterlašen möchte, da in seiner Beschreibung durch Di alt i besonder, hervorgehoben wird, daß die reichliche Schleimbildung nicht allein durch die normale Schleimhaut bewirkt, sondern durch eine Hyperplasic der Schleimhautgesteigertwird, die sogar zur Adenombildung führenkann. Auch bei Dialt 1 wird der außerordentlich chronisclie, symptomlose Verlauf besonders erwähnt - 30 Jahre. Auch her das Pseudo- 
myxom im Bru hsack cun Zutallsfund we in der Meluzahl der Fälle, bei denen allen selbst retrospektiv cin typischer klinischer Verlauf, etwa ein Anhaltspunkt für eine überstandene Appendicitis, nicht konstruiert werden konnte.

Eine der Hauptbedmgungen tur das Zuslandekommen eines Pseudomyxoms ist auch nach Dralti der aseptisch gewordene ciewebs $\%$ ustand nach Ablauf der Wurmfortsatzent. zündung, die zur Divert ikelbildung oder Abschnurung eines Appen. dixteiles führt, ein Umstand, der ja auch die klinische Symptomlosigkeit des samzen Verlaufs, die fast an allen . bbandlungen zur Sprache kommt, /l crklaren vermag. Auch in unserem 2. Falle war ja der Bruchsackinhalt steril und heilte die Operationswunde per primam.

Wiesen wir shon eingangs daraut hin, daB dic Appendix divertikelbildung pathologisch-anatomisch einen ,Heilungoprozel3” darstellen kann, andererseits ein I divertikel als locu minoris reststentiae oft und gern den Boden für eine rezidivierende Appen dicitis abgibt, so ist es auch erklarlich, dal3 der grobere Teil der beschriebenen Appendixdivertikel unter den klinischen Erschei nungen einer Appendicitis angegangen und entfernt wurde, der andere, geringere Toil Zufallsfunde darstelle. Fs wird uns dann aber auch verständlich, daß die bedingungen zur Bildung eines Pseudomyxoms viel seltener gegeben und wesentlich komplizierter sind. Der Entzündungsprozels, der zur Dirertikelbildung getühr hat, muß rollkommen zur Abheilungs gekommen, das entstandenc Divertikel bzw. scin Inhalt muli keimfrei geworden sem. Auch muli sich de. Entzindung in so mabigen Girenzen gehalten haben. daß nicht groBere Si heimhaupartien ler Appendix in ihrem ah. geschnürten Tesle zugrund: gehen, um imslande zu x'in, in lange claucruder Tätigheil seringen Sohleim zu produzieren, der das Divertikel, die schwachste Stelle der . Ippendix, zum Platzen bringt und hicrlurch den Zustand den J'seudomyxoma peritonei ex pro cessu vermiforme hervorrutt. Lis ist damn aber auch einleuchtend, daß das Pseudonyxoma peritonei in itial kaum klinische Symptome zu machen bratuht und spater ots nur mechaniach hinderlich in Erscheinung tritt.

Was nun noch dic Ausbreitung des lipithels in ater freien Bauchbrihle anhetriff. so ist einmal dic Möglichkeit gegeben, 
daß beim Platzen einer Divertikelwand überhaupt keine oder prak tisch keine Schleimhautepithelien mit versprengt werden, und daß das Appendixlumen die alleinige Quelle für die Schleimproduktion bleibt. Die Perforationsstellen der Divertikel werden ja of minimal klein angegeben.

So würden die Fraenkelschen und die ihnen ähnlichen meisten Fälle vielleicht zu deuten sein. Unbestritten durften aber die Oberndorferschen Funde wohlerhaltenen, schleimproduzierenden Schleimhautepithels auch außerhalb der Appendix in der freien Bauchhöhle sein, deren Existenzmöglichkeit natürlich um so besser gewährleistet ist, je weniger infektiös und entzündlich sich der ganze Prozel abspielt, die aber zweifellos cinen Bewein für die Implantationsfahigkeit von Appendix schleimhautepithel darstellen. Daf das verlagerte Epithel wucherungsfähig ist, Adenombildungen (Dialti) nachahmen oder papillomatöse Formen annehmen kann, ist nach den Untersuchungen unseres 2. Falles wohl einleuchtend, ebensu die Differenzierungsmöglichkcit in ein, fast nur aus Becherzellen bestehendes Zylinderepithel. Wie weit allerdings die Mutationsund Wachstumsfähigkeit dieser verlagerten Zellen geht, können wir nicht beantworten. Zwingende, uberzeugende Gründe aber gegen die Erklärung von Fällen wie den Merkels sind also nicht zu erbringen, so daß auch eine weitere Epithelausdehnung über größere Peritonealstrecken in den Bereich der Möglichkeit gezogen werden muß und deshalb Merkels Fall den ihm von dem Autor angewiesenen Platz als Pseudomyxoma peritonei ex processu vermiforme in der Literatur weiter behauptet. Auch der Comollesche Fall kann mit den eingeräumten Irrtumsmöglichkeiten dem Merkelschen Fall als Stütze dienen.

Während uns ohne weiteres einleuchtet, daß bei den Fällen wie denen Fraenkels, Neumanns. Honneckers und den ihnen ähnlichen initial klinische Symptome vollkommen fehlen können, ja selbst in fortgeschritteneren Stadien der Krankheit überhaupt nicht oder unbedeutend in Erscheinung treten, ist es doch als eigentümlich hervorzuheben, dal. sowohl Merkel als Co moll e ausdrücklich auf die Symptomlosigkeit und die Benignität des Krankheitsverlaufes auch ihrer Fälle hinweisen, was man 
nach dem pathologisch-anatomischen bilal nicht $\rightarrow$ ohne wences erwartet hätte. Es scheint also allen Fällen ron l'seudomyxoma peritonei, selbst denen, dic p:thologisch-anatomisch den nach geplatztem Urarialkystom vollkommen ähneln, die Geschwulstkachexic zu fehlen, die wir bei dem I'seudomyxom nach geplatztem ()arialkystom oft erwähnt finden. Das Krankheitsbild verläuft sehr langsam, braucht riele Jahre 7.4 seiner vollkommenen Ausbildung und zur Produktion nennenswerter Schleimmassen. Fand doch z. B. Oberndorfer immiten gar nicht einmal großer Schleimmengen wohlerhaltenes, rerlagertes Appendixschleimhautepithel, das nach seiner Ansicht clort schon jahrelang sich befand. Auch sind die Schleimmengen in Dialtis und meinem 2. Fall zuar absolut grob, als Produkt ciner 30-bzw. to jährigen Tätigkeit der obliterierten . Ippendix jedoch gering. Die Dauer des Bestehens des Pseudomyxoms in unserem I. Fall ist nicht annähernd anzugeben. Dal, das gelieferte Schleimprodukt selbst in großer Menge rein chemisch keinen Einflußs auf den Organismus hat, kcine Kachexie hervorruft, wild in allen Fällen hervorgehoben.

Ganz anders ist clas Bild bei dem I'seudomyxoma yeritonei als einer liolge eines geplatzlen Ovarialkystoms. Hicr bildet einmal den Ausgangspunkt ein 'Tumor, auch sheint dic 7.ellenaussat nach Ruptur des Kystons gleich manscuhaft tu sein. In den meisten Fällen zeigt sich klinisch ein Bild der malisnen (ieschwulstbildung. Oft wird Kathexicausbildung erw ̈̈hnt. Cirok ist die Neigung zun Rezidiv selbst nach Entfernum des Orarialkystoms, in den meisten Fällen in Kürze das schwere Krankheitsbild trotz "radikaler Operation" w ieder da, die meisten Befallenen in relativ kurzer Zeit zum Tode brmgend. Selten -nd die Ausgänge im Werthschen Sinne, Organjation den produzierten Schleims (und die Vernichtung des ihn lieferndeis Epithels). Den versprengten. auf allen Organen der Baulhbihle implantierten Epithelien des geborstenen () varialumor ist 'mu rege Schlemtätigkeit eigentümlich, zweifellow cine wesentlich intensivere als den verlagerten Appendixschleimhautzellen. In ein, zwei Jahren ist, trot $z$ radikaler Operation, die ganze Bauchhöhle wieder mit Schleim angefüllt. Es handelt sich also bei dem Pseudomyxoma peritonei als Folge eines geplatzten Ovarialkystoms um einen ganz anders gearteten, durchaun malignen Prozeb. Ei int das Verdient E. Fraenkels, bei 
der Abgrenzung dieser beiden rerschledenen Krankheitsbilder auch diesen Punkt hervorgehoben zu haben.

Nur in seltenen Fällen gelingt es also wirklich, bei dem l'seudomyxoma peritonei nach geplatztem Ovarialkystom radikal zu operieren. Wie traurig dementsprechend die operativen Heilerfolge sind, lehrte ein Blick in die einschlägige gynäkologische Literatur. Da es nun silt, die Schleimquellen zum Versiegen zu bringen, so wird man bei den meisten fällen von Pseudomyxoma peritonei ex processu vermiforme durch eine Appendektomie zum sicheren Ziele kommen, ob in allen Fällen, wie $z$. B. den von Merkel und ( $\mathrm{C}$ molle ähnlichen, entzieht sich bisher unserer Kenntnis. Theoretisch ist diese Frage ebensowenig zu beantworten, wie eine Urteilsbildung nach dem rein pathologischanatomischen Bild im Mikroskop moglich ist, wie groß die vitale Energic implantierten Appendixepithels bzw. Orarialkystomepithels ist. Wir wissen nur aus klinisch gezogenen Vergleichen, daß verlagertem Ovarialkystomepithel eine stärkere Proliferationi. und Produktionskraft innewohnt, als dem verlagerten Appendixschleimhautepithel. Ob Heilungen des Pseudomyxoma peritonei ex proressu rermitormi im Werthschen Sinne rorkommen können, Implantiertes Epithel clurch peritoneale Organisationsprozesse vernichtet werden kann, entzieht sich bisher unserer Kenntnis.

Die klinische Gutartigkeit aller bisher veröffentlichten Falle làbt für alle Fälle aussichtsrolle chirurgische Hilfe erhoffen.

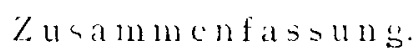

Die Appenduxdivertikelbildung ist kein seltener Befund. Vorwiegend einem entzïndlirhen Prozeß des Wurmfortsatzes verdankt es seine Entsteluung; nu selten ist es kongenitalen Ursprungs. Der Genese ceiner häufigsten Entstehung entsprechend durch Entzündung des Wurmfortsatzes gibt das Divertikel gern den Boden zu neuer Fntzündungen ab. Das Krankheitsbild dokumentiert sich dann klinisch unter den Zeichen der akuten oder chronischen rezidivierenden Appendicitı. Nicht allzu selten bildet das Appendixclivertikel als Produkt abgeheilter Wurmfortsatzentzündung einen Zufallsbefund bei Operationen und Obduktionen. Ein Folgezustand des Ippendixdivertikels bei central ierschlossenem $A_{p}$ pendixlumen ist das nog. Pseudomyxoma peritonei, für dessen $Z$ ur- 
standekommen die das Divertikel bildende Entzündung notwendig sur Ruhe gekommen, der Divertikelinhalt keimfrei oder nahezu keimfrei geworden sein muli.

Bei der Ruptur des Divertikels kann entweder nur brhleim in die freie Bauchhöhle entleert werden -- es bleibt dann die Appen dix allein die schleimspendende Quelle - oder es können außer Schleim- auch Schleimhautepithelien außerhalb des Appendixlumens in die freie Bauchhöhle implantiert werden und ihrerseits Schleim produzieren.

Die Schleimproduktion hält sich aber in mäßigen Grenzen, der Prozeß ist durchaus chronisch, lange Zeit bis zur vollen Ausbildung des Krankheitsbildes erheischend, fast durchwe: klinisch jahrelang symptomlos verlaufend. Die Natur des Pseudomyxoma peritonei ist in allen bisher beschriebenen Fällen gutartig, im Gegensatz zum Pseudomyxoma peritonei nach geplatztem Ovarialkystom, das im allgemeinen rasch und fast immer unter den klinischen Zeichen cines malisnen Tumors zum Tode fülurt.

\section{Literaturverzeichnis.}

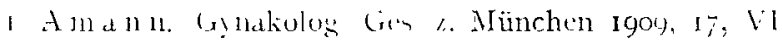

- Aschot 1 , De Wurmfortsatzentzundung Jena 1908

3. --., Pathogenese und Atiologic der Appondicitia. Ergebn d un Mecl. is

+ Borst. Geschwilste. 1 Teil

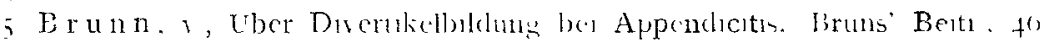
H. 3 .

1) Comolle. Frankfulte Zeltuche I Path. Bd. 19

7. Dialti, Pacudomixoma clel peritunes di orgene appendiculare Clen. Chir. IgI4, XXII. 9 Ref. Zentralbl. f Chir. 19I5.

8. Flbe. Brun' Beitr. I909. 64 (daselbst aungiebige Luteratuangabe)

9 Fracnkel. E. Münchner med. Wochenschr. Igor, Nr.24.

10. - Münchner med. Wochenschr. 1912, Nr.21 u. 22.

11. Graser. Verh. d. Deutsch Path. Ges Munchen is9g.

I2. Hansemann. V Virch. Archiv I896, Bd I4t.

13. He dinger. E. Virch. Archiv, Bd. 178, II. I, S. 25

if. Henke. Verh. d. Isutsch Pathul Ges. Numehen isgu

15. Hirt Anerican Obstetr. Journal LIII, p. 345

I6. Honecker. Frankfurter \%eitschr f Yalhol., +

17. H ü $\mathrm{ter}$. Zicglers Beitr., $4 \mathrm{I}$

18. Kirchborg. Uber cmigr -eitcne zysta he und karzmomatöse Tumoren cles Pcritoneums lirankfurter Zeits hr. f. Pathol., io.

19. Konjetan! Minchner med. Wochenschr, 1yog. S $225 \mathrm{r}$. 


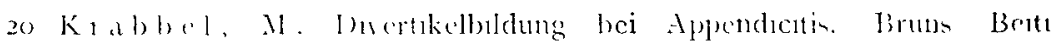
$1912,80$.

2 l.ejals et Ménétraer. Rér. de Chn 1004, $\mathrm{T}_{2}$

22 I cont fell. Ref. Zentralbl f. Chir 19r3. Nr. 4"

2.3 I. ubarsch Terh. d Deutsch. path. Ges 1900. Tahresturse t. aratl Fortbillg, Januar 19 r 4.

24 IIrkcl. Munchner med Wochenech. igut S ij6o und Verh d I) eutsch. Pathol Ges. Ioro, Erlangen

25 . Nertens. Grenzgeblete, Bd 9

2h, Michaclson, I'scudemyxoma peritemend Mannen Hygied IXXXVI! II 11 lief Zentralbl. f. Chrr. 19I5

27. $110 \mathrm{n}, \mathrm{k}$ cberg. Zieglers Beitrage 1903, it

28. II und. Ortherhe Festschrift Cottingen 1903

2) Neuma nn. Berlines kln Wochenschr. 1904, I

io. Wberndorfer. Verh. d. l)cutsch Pathol' Ge s. Igot und lubarsh (Istertag 1909, Bd. I3. I.

il Olshausen. Tie Kiankheten der (Marken $\$ 886$

32 Pfannenstacl. Veits Handb. d. Gynäkolugle r898. Bd 3

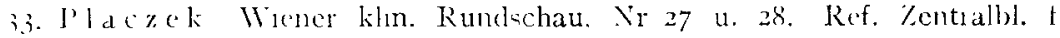
(hir. 191.3

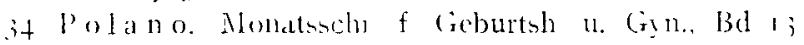

i5 Kehn. I' Fankfuter Ze:tscher f P'ath., Bol. 19

io Siman lentralbl 1. Chir. 1911, S 112

37. Sprengel. Appendictu Deutuche ('hu loget

38 Irotter Brit med. Toumal igro, s 68;

39 Writh Areh f iyn. 24. Si, rooft 